\title{
Systemsimulation als Teil des Systems Engineering für Scheinwerfer- und Pedalsysteme auf Basis der Modellbeschreibungssprache Modelica
}

\author{
Heinz-Theo Mammen ${ }^{1}$, Phillip Limbach¹, Thorsten Maschkio
}

${ }^{1}$ HELLA GmbH \& Co. KGaA

Rixbecker Straße 75, 59552 Lippstadt

\section{Kurzfassung.}

Im vorliegenden Beitrag wird anhand von zwei Anwendungsbeispielen gezeigt, welche Synergien mit Hilfe der Systemsimulation erzielt werden können. Beim ersten Beispiel mit Fokus auf die Temperaturentwicklung in einem DC-Steller, konnte der bisherige messtechnische Aufwand zur Identifizierung kritischer Betriebsfälle durch die Simulation um den Faktor 4 reduziert werden. Das zweite Beispiel, ein Bremspedalsystem, zeigt eine Möglichkeit der Kontaktmodellierung zwischen starren Körpern, um Bewegungsabläufe zwischen diesen Körpern beschreiben zu können. Durch dieses neue Verfahren können Körperkontaktflächen auf einfache Weise in Modelica [1] beschrieben werden, wodurch der ursprüngliche Modellierungsaufwand für Kontaktflächen um den Faktor 10 reduziert werden konnte.

Mit Hilfe der Simulationsmodelle konnte das jeweilige Designkonzept optimiert sowie das Reib- und Temperaturverhalten einzelner Komponenten entsprechend den Spezifikationen angepasst werden.

\section{Einleitung}

Auf Grund der wachsenden Komplexität von Systemen und Subsystemen im Automotiv-Bereich gewinnt das Thema Systemsimulation als integrativer Bestandteil des Systems Engineering immer mehr an Bedeutung, um die stetige Absicherung des interdisziplinären Systemverhaltens über alle Phasen des Produktentstehungsprozesses hinweg von der Problemstellung bis zur Produktion zu gewährleisten. Im Rahmen der Systemsimulation bei Hella wurden in den vergangenen Jahren Systemmodelle für unterschiedliche Komponenten aus dem Automobilsektor entwickelt und validiert. Hierzu zählt unter anderem ein Modell eines DC-Stellers eines mechatronischen Scheinwerfermoduls mit den Komponenten Ansteuerung, elektrische Verstelleinheit und Getriebe unter Berücksichtigung des Umwelteinflusses Temperatur sowie ein Pedalsystem mit den Komponenten Gelenksystem, Positionserkennung sowie Rückstellmechanismus. Beim Pedalsystem wurde die Modellgrundstruktur automatisch aus dem Geometriedesign mit integrierter Kine- matik heraus generiert und um Komponenten zur Beschreibung des Reibverhaltens, der Fußkraft, der Rückstellfedern sowie der Kontaktflächen erweitert.

Die Entwicklung der Modellstrukturen inklusive der Modellierung von Kontaktflächen erfolgte auf Basis der Modellierungssprache Modelica und dem Simulator Dymola [2]. Im Fokus dieses Beitrags liegen die spezifischen Eigenschaften der Modelle inklusive der Kontaktmodellierung sowie die automatische Modellgenerierung via direkter Kopplung zwischen Design- und Systemsimulationswerkzeug.

\section{Modell eines DC-Stellers}

Der Trend zu immer mehr mechatronischen Komponenten im Fahrzeug ist beispielhaft am Scheinwerfer erkennbar. Wo früher Leuchtmittel mit Reflektor in einem einfachen Gehäuse für eine Ausleuchtung der Straße gesorgt haben, treten heute komplexe mechatronische Scheinwerfersysteme, die ein dynamisches Kurvenlicht beinhalten, die über eine automatische Leuchtweitenregelung verfügen und die je nach Fahrstrecke (innerorts, Landstraße, Autobahn) für eine angepasste Ausleuchtung sorgen.

Da diese mechatronischen Systeme immer komplexer werden, ist eine ganzheitliche Entwicklung sinnvoll und notwendig. Unterstützt werden kann diese Entwicklung durch eine entwurfsbegleitende Modellierung von der Konzeptphase bis zum Serienbaustein.

Der DC-Steller ist eine Teilkomponente des mechatronischen Systems Scheinwerfer. Diese Teilkomponente stellt für sich auch bereits ein mechatronisches System bestehend aus einer Ansteuerung, einer elektrischen Verstellkomponente und einem Getriebe inklusive Verstellarm dar. Bild 1 zeigt einen DC-Steller eines mechatronischen Scheinwerfermoduls.

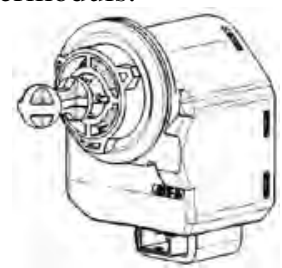

Bild 1: DC-Steller eines mechatronischen Scheinwerfermoduls 
Im Folgenden werden die einzelnen Modellkomponenten detailliert beschrieben.

\subsection{Ansteuerung des DC-Stellers}

Die Ansteuerung des DC-Stellers ist in Bild 2 dargestellt. Sie besteht im Wesentlichen aus zwei Halbbrücken und einer Logik zur Ansteuerung dieser Halbbrücken.

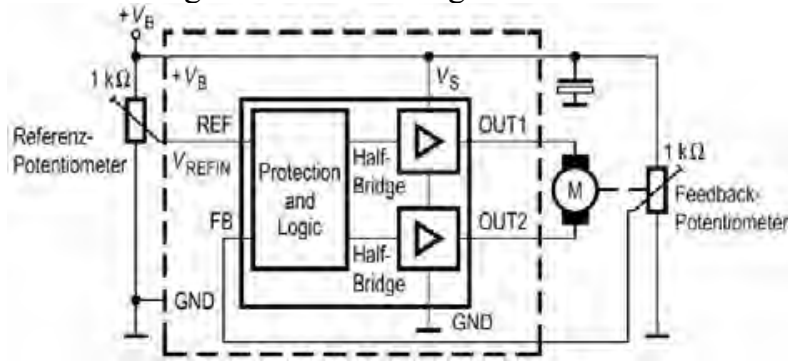

Bild 2: Ansteuerung eines DC-Stellers

Das zur Ansteuerung eines DC-Stellers entwickelte Modell ist in Bild 3 dargestellt. Es wurde hierarchisch modelliert, d.h. die in Bild 2 dargestellten Halbbrücken z.B. sind Teil des ICs TLE4209G.

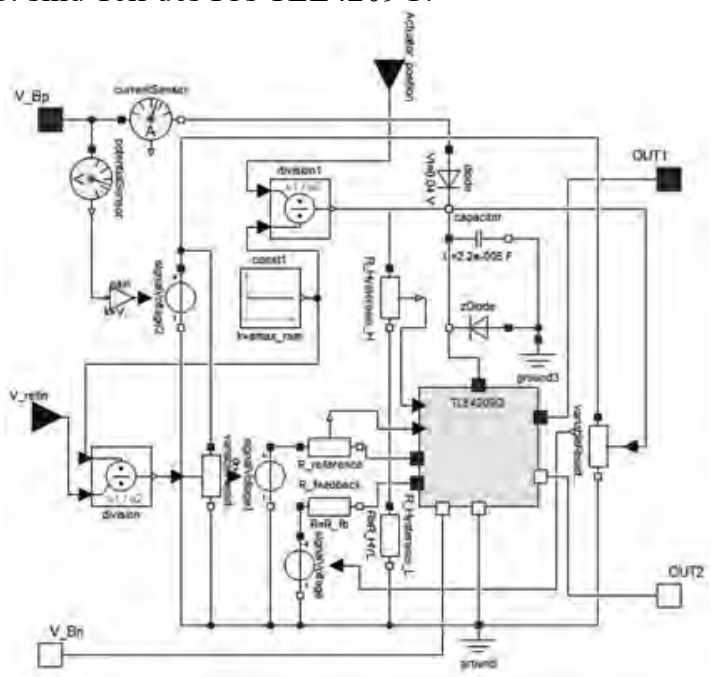

Bild 3: Modell der Ansteuerung eines DC-Stellers

\subsection{Motormodell des DC-Stellers}

Das Motormodell beschreibt das elektrische und mechanische Verhalten eines DC-Motors. Weiterhin wird mit dem Modell das thermische Verhalten nachgebildet, d.h. es wird einerseits die Umgebungstemperatur des Motors berücksichtigt und andererseits die Eigenerwärmung im Betriebsfall. In

Bild 4 ist das Modell des DC-Motors als Dymola/Modelica Beschreibung dargestellt.

Um das thermische Verhalten des DC-Motors richtig modellieren zu können, muss das Kommutierungsverhalten zunächst beschrieben werden. Der Motor besteht aus drei Wicklungen, die über einen Kommutator bestromt werden. Im Blockierfall (Störungsfall) muss der Motor eine kontinuierliche Bestromung über einen längeren Zeitraum unbeschadet überstehen. Dabei steigt die Motortemperatur auf Grund der Eigenerwärmung relativ schnell je nach Blockierung auf über $200^{\circ} \mathrm{C}$ an, so dass in vielen Fällen die kritische Motortemperatur überschritten wird. Dies führt zum Ausfall des Stellers.

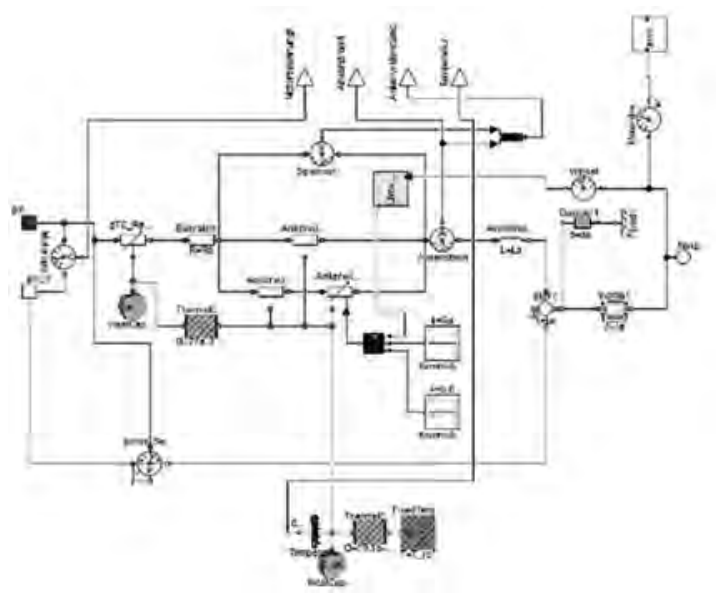

Bild 4: Motormodell des DC-Stellers

Die Simulation soll zeigen, wie sich das Temperaturverhalten verschiedener Motoren im Blockierfall verhält. Dabei wird zwischen den zwei Blockierfällen, der 1/3und der 2/3-Blockierung, unterschieden. Beim 1/3-Fall stehen beide Bürsten jeweils auf einer Lamelle des Kommutators, im 2/3-Fall steht eine der beiden Bürsten zwischen zwei Lamellen, wodurch diese überbrückt werden. Damit kommt es zu den folgenden zwei Ankerwiderstandszuständen im Blockierfall:
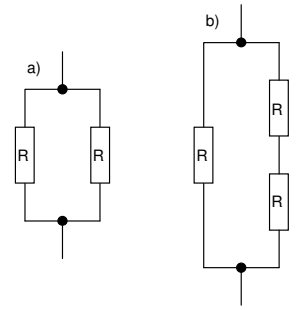

\section{Bild 5: a) 2/3-Blockierfall; b) 1/3-Blockierfall}

Im 2/3-Fall beträgt der Gesamtankerwiderstand Rges=1/2 R und im 1/3-Fall Rges=2/3 R. Diese beiden Fälle wurden im Modell gesondert einstellbar modelliert, um das Temperaturverhalten im Blockierzustand für diese Fälle explizit ermitteln zu können.

\subsection{Getriebemodell des DC-Stellers}

Die Getriebekomponente beschreibt unter anderem das Verhalten eines Schneckengetriebes sowie die Anschläge für den Hin- und Rücklauf des Stellers. Zudem werden mit diesem Element Reibparameter, wie z.B. die Coulomb- und die Stribeck-Kraft modelliert. Somit ge- 
hören zu den Parametern, die mit dieser Modellkomponente modelliert werden, das Übersetzungsverhältnis, die beiden Anschläge und die Reibung. In Bild 6 ist das Modell der Getriebekomponente dargestellt.

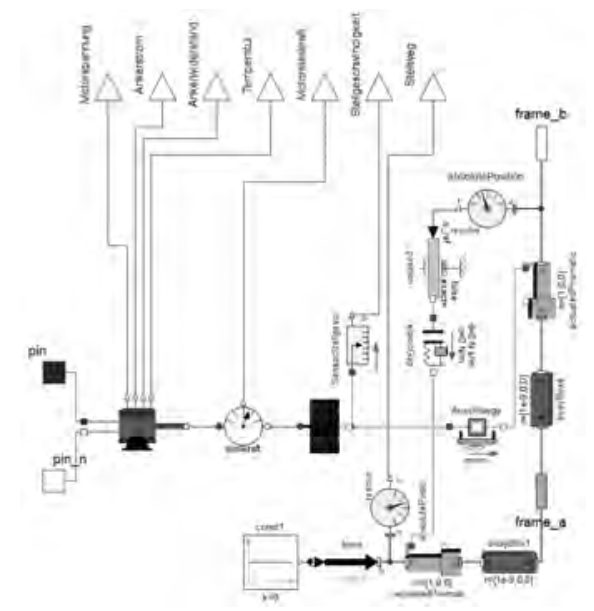

Bild 6: Getriebekomponente des DC-Stellers (inklusive Motor)

\subsection{Modellvalidierung}

Neben der Modellierung spielt die Modellvalidierung eine wichtige Rolle, damit am Ende des Prozesses ein Modell zur Verfügung steht, das für Untersuchungen während des gesamten Entwicklungspfades von der Konzeptidee bis zum fertigen Produkt ,uneingeschränkt“ genutzt werden kann.

Die Parametrisierung eines Modells kann auf Basis von Datenblattangaben oder anhand von Messungen erfolgen. Speziell bei Motoren sind die Datenblattangaben häufig nicht ausreichend, um alle Parameter definieren zu können. Daher sind Messungen notwendig, die oft sehr aufwendig und kompliziert sein können und es wird dafür spezielles Messequipment benötigt.

Im vorliegenden Fall konnten die wesentlichen Motorparameter über Datenblattangaben berechnet werden, die Temperaturparameter wurden mit Hilfe von Messungen unter anderem im Klimaschrank ermittelt. Dabei wurden Untersuchungen zu den drei Betriebsfällen Drehbewegung, 1/3- und 2/3-Blockierfall durchgeführt.

Auf Basis der Untersuchungsergebnisse wurden die Modellparameter berechnet und ins Modell eingetragen. Nachdem alle Parameter bestimmt sind, ist es notwendig, die Funktion des Modells anhand ausgewählter Testuntersuchungen zu überprüfen. Dazu werden entsprechende Testanordnungen erstellt und die mit diesen Anordnungen erzielten Simulationsergebnisse mit Messungen verglichen. In Bild 7 ist eine entsprechende Testanordnung dargestellt.

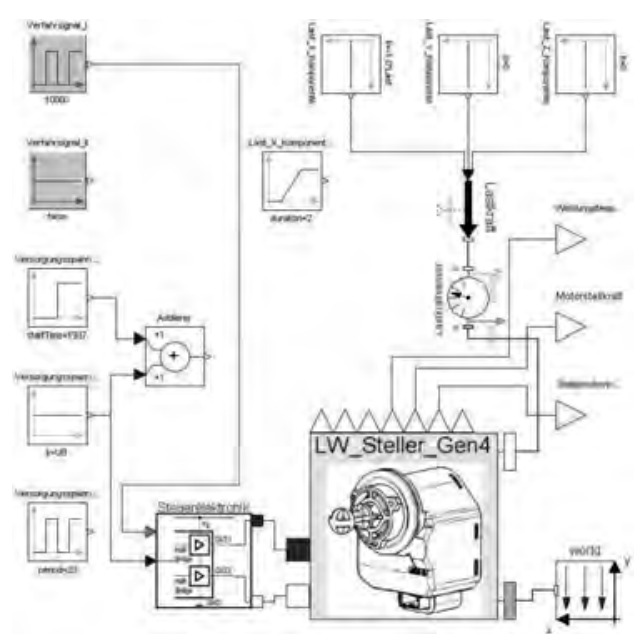

Bild 7: Testanordnung zur Validierung des Modellverhaltens

In Bild 8 ist beispielhaft ein Vergleich zwischen Messung und Simulation dargestellt. Es wurde der Ankerstrom des DC-Motors ermittelt.

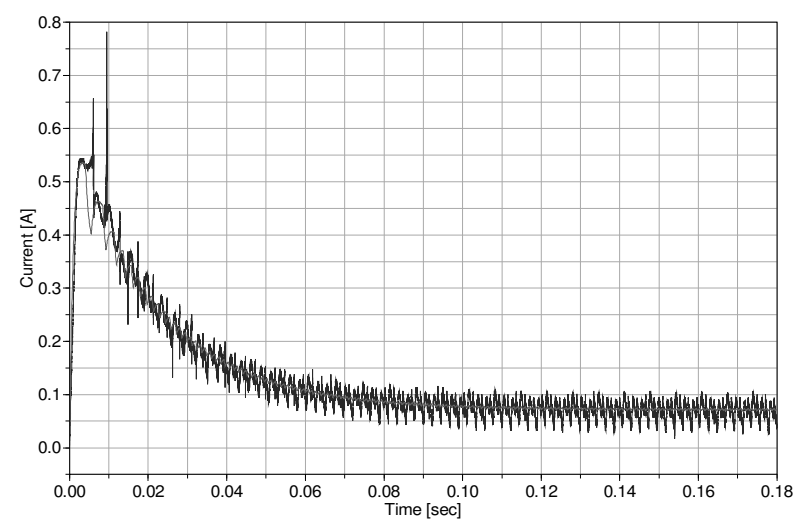

Bild 8: Ankerstrom des DC-Motormodells - Vergleich Messung (blau)/Simulation (rot)

Der Vergleich zwischen Messung und Simulation zeigt eine gute Übereinstimmung (weitere Simulationen haben dieses Ergebnis bestätigt). Damit kann dieses Modell für Untersuchungen im Entwicklungsbereich eingesetzt werden.

Die Analyse des Aufwandverhältnisses zwischen der Prototyp-Hardware-Entwicklung und der Modellierung/Simulation ergibt, dass das Verhältnis für das obige Beispiel bei 4:1 liegt, d.h. der Aufwand für die HardwareEntwicklung ist $4 \mathrm{mal}$ so hoch. Mit der Modellierung/Simulation kann der Entwicklungsaufwand für einzelne Konzeptphasen also signifikant reduziert werden. 
Im Fahrzeug werden drei verschiedene Arten von Pedalsystemen unterschieden: das Bremspedal, das Kupplungspedal sowie das Gaspedal. Da diese Pedalsysteme nicht mehr wie vor Jahren direkt mit den zu betätigen Systemen verbunden sind (z.B. das Gaspedal direkt über ein Seilsystem mit dem Motor), werden bei den heutigen Pedalsystemen andere Anforderungen ans Pedal gestellt. Daher sind die heutigen Pedalsysteme neben dem Betätigungsarm mit Reibelementen, Sensoren und Zusatzfedern ausgestattet, um das ursprüngliche haptische Verhalten so gut wie möglich nachzubilden.

Ein Beispiel eines Pedalsystems ist in Bild 9 dargestellt.

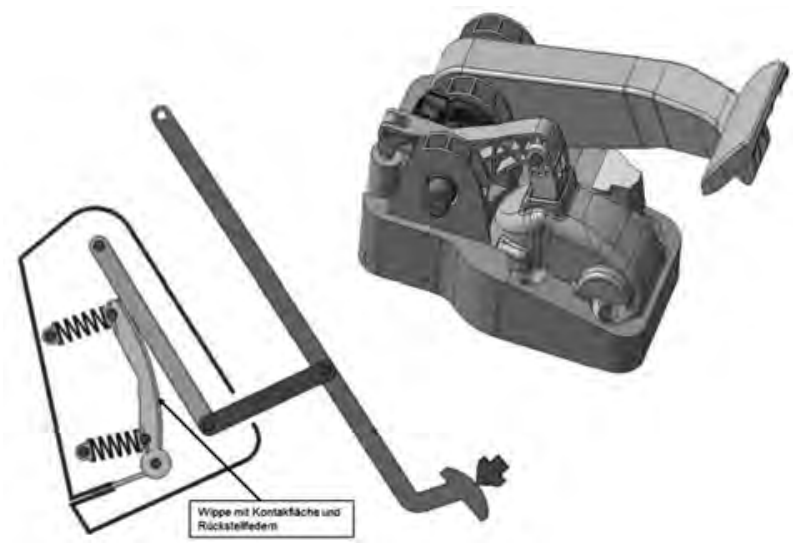

Bild 9: Designbeispiel eines Bremspedals

Das in Bild 9 dargestellte Bremspedal besteht im Wesentlichen aus einem Viergelenk, einer Wippe inklusive Rückstellfedern, einer Kontaktfläche und einem Reibelement. Die Kontaktfläche bildet die Schnittstelle zwischen dem Viergelenk und der Wippe. Mit Hilfe der geometrischen Form der Kontaktfläche läßt sich das Kraft-Weg Verhalten des Pedals entsprechend der Kundenspezifikation (siehe Bild 13) verändern.

Die wesentlichen Elemente des Pedals lassen sich mit Hilfe von Modelica-Standardelementen modellieren. Die Modellierung einer Kontaktfläche stellt eine besondere Herausforderung dar, daher wird im Folgenden darauf näher eingegangen. Eine Möglichkeit, um Kontaktvorgänge in Dymola zu simulieren, bietet die Idealized Contact Library [3].

\subsection{Beschreibung der Oberfläche einer Kontaktfläche}

In der Systemsimulation werden Körper meist als ideal starr angenommen. Diese Annahme ist möglich, da die Betrachtung der Kinematik im Vordergrund steht. Hierfür ist es ausreichend, die Körper durch die Lage des

Schwerpunkts sowie durch die Masse und die Trägheitsmomente im Schwerpunkt zu beschreiben. Die Ausdehnung der Körper wird dabei nicht weiter betrachtet. Für Kontaktphänomene ist jedoch sowohl die Modellierung von elastischen Körpern als auch die Definition der Oberfläche zwingend notwendig.

In der Idealized Contact Library steht für die Beschreibung von einfachen Geometrien, wie einem Rechteck, einem Zylinder oder einer Kugel, jeweils ein „surface“- Block zur Verfügung, der die Dimensionen der Oberfläche sowie deren Ausrichtung im angebundenen Koordinatensystem beschreibt. In der neuesten ReleaseVersion sind zusätzlich Blöcke für die Beschreibung von Ellipsoiden sowie anderer konvexer Körper implementiert. Der „surface“- Block stellt eine dünne, masselose Fläche dar, die an jeden Starrkörper über eine „frame““Schnittstelle angebunden werden kann.

Die eigentliche Berechnung der Kontaktkraft findet im ,contact"- Block statt. Die notwendigen Informationen über die Kontaktoberfläche werden dabei über eine „contact"- Schnittstelle übermittelt. Neben der Definition des körperfesten Koordinatensystems der Oberfläche, werden auch die geometrischen Informationen der Kontaktfläche sowie in der neusten Release-Version die Information über den Oberflächentyp transferiert.

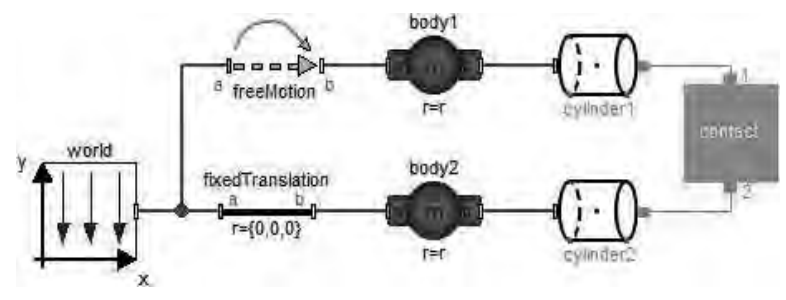

\section{Bild 10: Aufbau eines einfachen Kontaktmodells mit zwei Zylindern}

In Bild 10 ist ein Beispielmodell für zwei in Kontakt tretende Zylinder dargestellt. Die als „cylinder1“ und „cylinder2“ bezeichneten Blöcke beschreiben die Oberfläche, die durch einen ,,contact“- Block (orange) verbunden sind. Komplexere Geometrien können durch Parallelschaltung einzelner Kontaktoberflächen zusammengefügt werden, wobei jedes Kontaktpaar über einen „,contact“- Block verbunden sein muss [3].

\subsection{Oberflächenmodellierung durch analytische Funktionen}

Der Ansatz, die Kulissenoberfläche durch analytische Funktionen zu beschreiben, entspringt aus der Notwendigkeit einer krümmungsvariablen Oberfläche. Diese soll über den betrachteten Bereich tangential- und krümmungsstetig sein. 


\section{Polynomfunktionen dritten Grades}

In diesem Ansatz wird die Kulissengeometrie durch eine oder mehrere Polynomfunktionen beschrieben, die im Schnittpunkt die erforderlichen geometrischen Bedingungen erfüllen. Definiert man eine Polynomfunktion dritten Grades mit

$$
\begin{array}{r}
f(z)=a_{3}\left(z-z_{0}\right)^{3}+a_{2}\left(z-z_{0}\right)^{2} \\
+a_{1}\left(z-z_{0}\right)+a_{0}
\end{array}
$$

so lässt sich die Funktion, nach Bestimmung der Koeffizienten $a_{i}$ sowie des Parameters $z_{0}$, eindeutig beschreiben. Sind die Koordinaten von zwei Punkten auf der Funktion sowie die erste und zweite Ableitung in einem Punkt bekannt, lassen sich die unbekannten Variablen durch

$$
\begin{aligned}
& a_{0}=f\left(z_{1}\right)-a_{1}\left(z-z_{0}\right)-a_{2}\left(z-z_{0}\right)^{2} \\
& -a_{3}\left(z-z_{0}\right)^{3} \\
& a_{1}=\frac{f\left(z_{2}\right)-a_{0}-a_{2}\left(z-z_{0}\right)^{2}-a_{3}\left(z-z_{0}\right)^{3}}{\left(z-z_{0}\right)} \\
& a_{2}=\frac{f^{\prime}\left(z_{1}\right)-a_{1}-3 a_{3}\left(z-z_{0}\right)^{2}}{2\left(z-z_{0}\right)} \\
& a_{3}=\frac{f^{\prime \prime}\left(z_{1}\right)-2 a_{2}}{6\left(z-z_{0}\right)}
\end{aligned}
$$

berechnen. Der Parameter $z_{0}$ wird gleich Null gewählt.

Soll die Polynomfunktion eine weitere Funktion in einem Punkt tangential- und krümmungsstetig schneiden, werden die erste und zweite Ableitung im Schnittpunkt durch die bereits definierte Funktion vorgegeben. Hierbei ist lediglich die Position der beiden Punkte zu definieren. Das Modell zur Beschreibung einer Polynomfunktion wurde als Modelica Modell abgebildet. Ein Teil des Kontaktflächenmodells, die Modellkomponente zur Berechnung des Kontaktpunktes auf der Ellipsenoberfläche ist in Bild 11 dargestellt.

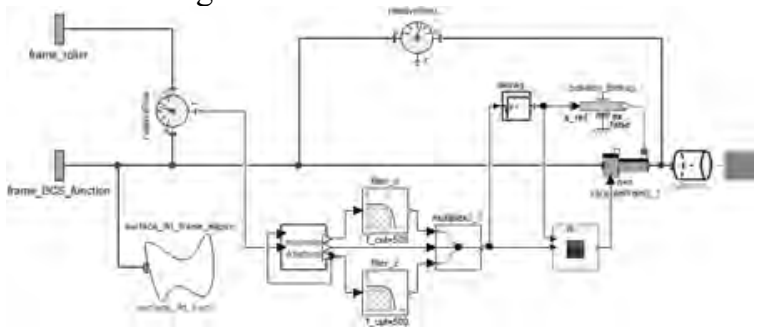

Bild 11: Modell zur Berechnung des Kontaktpunktes auf der Ellipsenoberfläche

Für die Berechnung des Kontaktpunktes auf der Kulissengeometrie wurde das Newton-Verfahren implementiert.

Ist die Polynomfunktion für einen bestimmten Bereich definiert, ist die Berechnung des minimalen $\mathrm{Ab}$ standes nur innerhalb dieses Bereiches notwendig und teilweise nur hierin möglich. Aus diesem Grund wird durch vorab definierte Grenzwerte die Berechnung des minimalen Abstandes auf den Definitionsbereich eingeschränkt. Programmtechnisch wird dies durch Abfrage des berechneten Kontaktpunktes gelöst. Liegt dieser außerhalb des Bereiches, wird er gleich dem definierten Grenzwert gesetzt.

Die Kulissengeometrie lässt sich nicht durch eine einzelne Polynomfunktion beschreiben, die das gewünschte Pedalverhalten über den gesamten Pedalweg generiert. Deshalb wird das Modell sukzessiv durch mehrere Funktionen aufgebaut. Dies geschieht im ständigen Abgleich mit dem gewünschten Pedalverhalten.

\section{Realisierung der Kulissengeometrie}

Im ersten Schritt wird die Kulissenform, die zunächst durch eine einzelne Polynomfunktion beschrieben wird, entsprechend der vorgegebenen Vorspannkraft und der implementierten Wippenfederparameter derart angepasst, dass die Pedalkraft zu Beginn der Auslenkung dem Wunschverhalten entspricht. Weicht die Kraft bei größeren Pedalwinkeln von den Referenzwerten ab, wird die Position des Kontaktpunkts bei dem entsprechenden Pedalwinkel auf der Funktionsoberfläche bestimmt und als Schnittpunkt zur weiteren Funktion definiert. Im nächsten Schritt wird der zweite Punkt der ,anschließenden“ Funktion derart gewählt, dass das gewünschte Pedalverhalten für einen weiteren Bereich erreicht wird. Dies wird sukzessiv durchgeführt bis die Kulissengeometrie vollständig modelliert ist.

Auf Basis der auf diese Weise definierten Kulissengeometrie kann im nächsten Schritt die ermittelte Oberflächenform direkt in CATIA [4] beim Design der Geometrie berücksichtigt werden. Hierdurch können Konstruktionsschritte und Prototypentwicklungen inklusive Messungen reduziert werden.

\subsection{Validierung des Gesamtmodells}

Das in Bild 12 dargestellte Gesamtmodell setzt sich im Wesentlichen aus drei Hauptkomponenten zusammen.

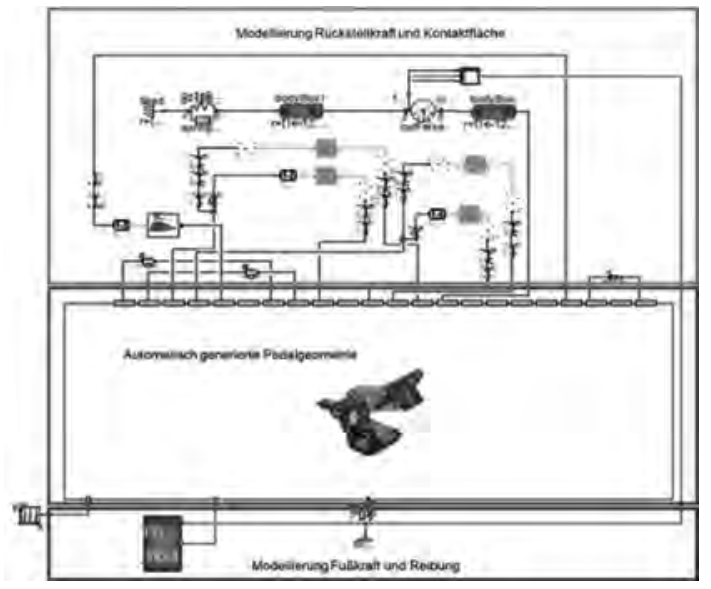

Bild 12: Gesamtmodell des Pedalsystems 
Hierbei handelt es sich um die direkt aus dem CATIA Design generierte Pedalgeometrie, einer Komponente zur Modellierung der Rückstellkraft und der Kontaktfläche zwischen dem Pedalviergelenk und der Wippe sowie einer Komponente zur Beschreibung der Fußkraft und der Reibung, die im Pedal auftritt.

Das mit diesem Modell erzeugte Simulationsergebnis ist in Bild 13 dargestellt. Es zeigt den Verlauf der Pedalkraft (rot) über den gesamten Pedalweg im Vergleich zur Wunschkennlinie.

Beim Rücklauf weicht das Simulationsergebnis unter den gewählten Parameterbedingungen vom Wunschverhalten ab. Dennoch ist der tendenzielle Verlauf ähnlich dem gewünschten Verhalten. Durch Erhöhung des gewählten Reibwertes, der im Modell angenommen und nicht durch reale Messungen verifiziert ist, wird die Spreizung der Hysteresekennlinie vergrößert. Unter Anpassung der Kulissengeometrie, lässt sich somit das gewünschte Rückstellverhalten nahezu abbilden, wobei die Rückstellkraft bei einem Pedalwinkel von $32^{\circ}$ unterhalb des geforderten Kraftminimums liegt. Hingehend zu einem geringeren Pedalwinkel, wird das Wunschverhalten in der Simulation annähernd erreicht.

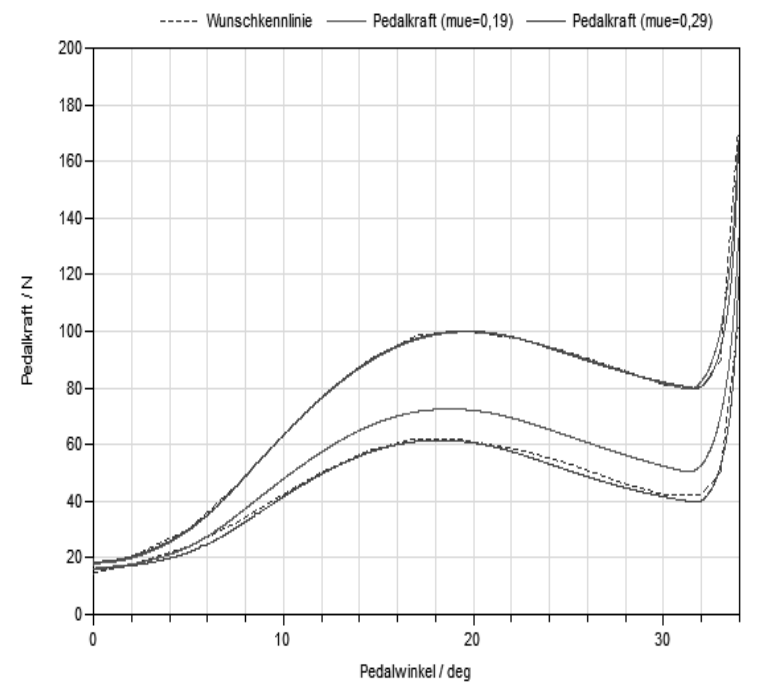

Bild 13: Einfluss der Pedalkraft bei Veränderung des Reibwertes

\section{Zusammenfassung}

Die Systemsimulation birgt viele Vorteile, da über alle Produktentstehungsphasen hinweg von der Problemdefinition bis zur Produktion eine zusätzliche Absicherung erfolgt, Kosten eingespart (z.B. weniger Prototypen), Zeitaufwände reduziert und Aussagen zu Systemeigenschaften getroffen werden können. Letztere wären mit Hardware-Aufbauten nicht oder nur mit erheblichem Aufwand möglich (z.B. Kraftmessungen in einem gekapselten System).
Die Modellbeispiele haben gezeigt, dass mit der Simulation Kundenanforderungen schnell überprüft, kritische Betriebsfälle simulativ abgebildet sowie Parameterund Aufbaustudien vereinfacht durchgeführt werden können. Die dabei gewonnenen Erkenntnisse tragen zu einem umfassenderen Systemverständnis bei. Entwicklungsfehler in frühen Entwicklungsphasen, die fatale Folgen haben können, lassen sich auf diese Weise meist vermeiden.

Ein weiterer Vorteil der Systemsimulation ist in der Reproduzierbarkeit der Versuchsbedingungen zu sehen. Kraft- und Bewegungsszenarien können z.B. durch Messungen nicht exakt reproduziert werden. Insbesondere für die spätere Testphase ist dies ein wichtiger Punkt.

Ein nicht zu unterschätzender Aspekt ist auch die Möglichkeit zur Visualisierung von Bewegungsabläufen mittels Animation. Durch Animation lässt sich das Systemverhalten in seiner Gesamtheit „erleben“ und bewerten. Dies gewährleistet eine schnellere Ergebnisinterpretation und erleichtert überdies die Kommunikation zwischen den beteiligten Entwicklern. Voraussetzung hierfür ist der Aufbau eines Mehrkörpersystemmodells, mit dem unter anderem der mechanische Aufbau nachgebildet werden kann.

Bezogen auf den Entwurfsablauf lassen sich Systemmodelle auch als ausführbare Lastenhefte auffassen. Auf diese Weise dokumentiert sich der Entwicklungsprozess teilweise von selbst. Alle Experimente lassen sich auch im Nachhinein reproduzierbar nachvollziehen. Dies erleichtert den Nachweis von Fehlerursachen und vereinfacht überdies die nachträgliche Durchführung von Änderungen.

In der Konzeptphase geht es unter anderem darum, die in Frage kommenden Konstruktionsvarianten untereinander $\mathrm{zu}$ bewerten, um das bestgeeignete Lösungskonzept zu ermitteln.

\section{Literaturverzeichnis}

[1] Fritzson, P.: Object-Oriented Modeling and Simulation with Modelica 2.1. John Wilex \& Sons, New York 2004.

[2] DYMOLA: Multi-Engineering Modeling and Simulation. www.dynasim.se, www.dymola.com.

[3] Oestersötebier, F.; Wang, P.; Trächtler, A.: A Modelica Contact Library for Idealized Simulation of Independently Defined Contact Surfaces. Paderborn.

[4] Dassault Systemes: 3DExperience Platform https://www.3ds.com/de/ueber-3ds/3dexperienceplattform/ 